\title{
An optical wireless system replacing cabled connections for the data transmission in the AIT phase
}

L. Gilli, G. Cossu, M. Rannello, A. Messa, E. Ciaramella

L. Gilli, G. Cossu, M. Rannello, A. Messa, E. Ciaramella, "An optical wireless system replacing cabled connections for the data transmission in the AIT phase," Proc. SPIE 11852, International Conference on Space Optics ICSO 2020, 118525T (11 June 2021); doi: 10.1117/12.2600013

SPIE Event: International Conference on Space Optics - ICSO 2021, 2021, Online Only 


\section{International Conference on Space Optics-ICSO 2020}

Virtual Conference

30 March-2 April 2021

Edited by Bruno Cugny, Zoran Sodnik, and Nikos Karafolas
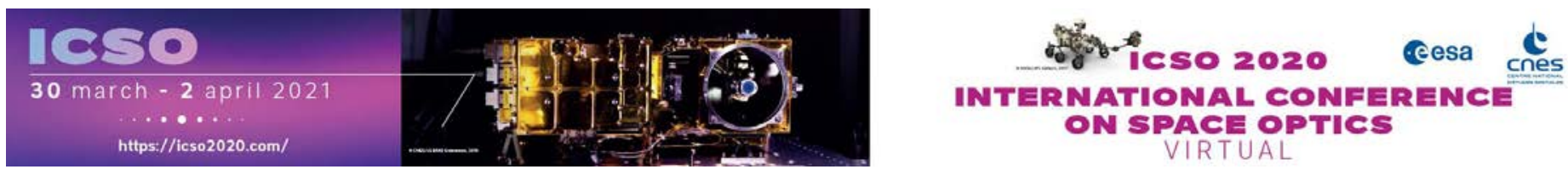

\section{An optical wireless system replacing cabled connections for the data transmission in the AIT phase}

\section{Cesa isoporecestings denes}




\title{
An optical wireless system replacing cabled connections for the data transmission in the AIT phase
}

\author{
Gilli L. ${ }^{a}$, Cossu G. ${ }^{\text {, }}$ Rannello M. ${ }^{\text {a }}$, Messa A. ${ }^{\text {, }}$, and Ciaramella E. ${ }^{a}$ \\ aScuola Superiore Sant'Anna, Via Giuseppe Moruzzi 1, Pisa, Italy
}

\begin{abstract}
Nowadays, one of the key issues when designing a Spacecraft (S/C) is to accommodate bundle of wires. The harness in the traditional wired communication poses relevant problems during the Assembly, Integration and Test (AIT) phase. Therefore, the space industry is actively considering wireless solutions for next satellite missions. A solution to these issues can be represented by the Optical Wireless Communication (OWC) systems. They show many advantages over traditional wired communication and wireless RF systems, which include reduction of harness mass, electrical isolation and immunity to Electro-Magnetic Interference (EMI). Recently, the European Space Agency (ESA) funded a project to prove the implementation feasibility of the OWC for intra-S/C, extra-S/C and AIT. The last scenario is expected to be the first to adopt OWC, thanks to the lowest risks associated with the choice of this application scenario. Here we present and demonstrate a new OWC system design for typical AIT scenarios. The system implements a bidirectional communication between S/C devices to the Electrical Ground Support Equipment (EGSE), able to replace the MIL-STD-1553B connection cables. This is achieved by means of optical transceivers realized exploiting only low-cost and Commercial Offthe-Shelf (COTS) components. Here, the characterizations of the Transceiver (TRX) results are reported, the measured receiver sensitivity is compatible with expected received optical power in the AIT scenario. Therefore, the systems performance was tested transmitting a MIL-STD-1553B signal. We also measured the received optical power and calculated the link margin.
\end{abstract}

\section{INTRODUCTION}

During a space program, an essential stage is devoted to the Assembly, Integration and Test (AIT) operations. The AIT is a key phase in the development of new Spacecrafts (S/Cs) and is carried out with the aim of testing both the individual components and subsystems, and the entire $\mathrm{S} / \mathrm{C}$ in order to verify their correct functionality (Ref.1). Therefore, these operations are performed to ensure the success of the launch phase and the proper execution of the S/C tasks. The AIT phase may last from a few days to a few years, depending on the satellite mission, the $\mathrm{S} / \mathrm{C}$ dimension and the complexity of the system.

Wireless solutions for data transmission would have great advantages in carrying out the AIT operations, reducing both the integration setup time and the tests execution. Moreover, the AIT phase turns out to be an excellent candidate for the wireless link application development; these activities do not involve the costs and timings associated to the $\mathrm{S} / \mathrm{C}$ launch, which allow to have low risks associated to the test of new technologies (Ref.2). Developing and testing these Optical Wireless (OW) systems can also be convenient in subsequent phases (e.g. on board S/C applications). The OW can help simplify the system significantly, allowing reducing the bulk and weight due to shielding and wires that, in the traditional wired communication, represents up to around $10 \%$ of the total satellite mass (Ref.2). Therefore, wireless solutions allow to establish high bit rate transmission without an increase of harness mass. This lower weight reduces the use of fuel, making the wireless technologies advantageous from an economic point of view.

Radio Frequency (RF) wireless technologies (e.g., WiFi) can be a good solution, simplifying significantly the communication harness (Ref.3 - 4). However RF wireless links introduce strong constraints in terms of security and Electro-Magnetic Compatibility (EMC). In presence of RF emissions, the electronic equipment could be subject to susceptibility issues that can cause malfunctions and damage to the equipment.

Further author information: (Send correspondence to Lorenzo Gilli)

Lorenzo Gilli.: E-mail: lorenzo.gilli@santannapisa.it 


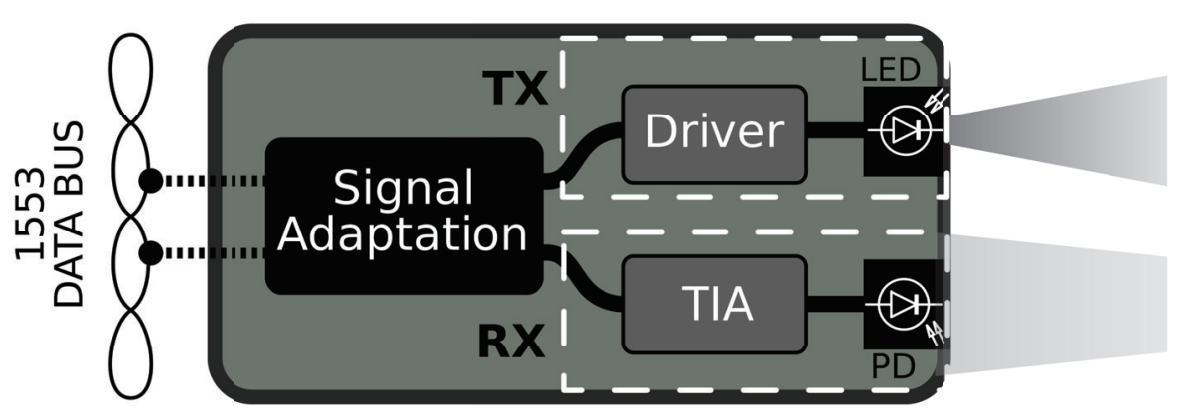

Figure 1: AIT transceiver (TRX). On the left, dotted lines highlight the connection between the electrical MIL-STD-1553B Data Bus and the signal adaptation circuit. On the top right, we have the TX (LED and driver circuit) and on the bottom right RX (PD and TIA circuit). The continuous lines highlight the electrical connection between TX/RX and signal adaptation board, for the single-ended signal transmission.

A solution to these issues can be represented by the Optical Wireless Communication (OWC) systems (Ref. 5 - 6). These communication technologies are recently emerging as a promising alternative to RF communication in those scenarios where RF systems are limited, both from physical and legislative restrictions. They show many advantages over traditional wired communication and RF systems, which include reduction of harness mass, electrical isolation and absence of Electro-Magnetic Interference (EMI) sources. OWC systems are very versatile and they are usable in a large range of different situations. Depending on the application, this technology is available at different values of data rate, link distances and energy consumption. Using a laser based setup, it is possible to reach high bit rate in the order of tens of Gbit/s (Ref. 7 - 8 - 9). Lower data rate (tens of Mb/s) OWC applications were also developed. These configurations usually use Light Emitting Diodes (LEDs), as they are optical sources with lower cost and higher optical power, and photo-detectors with large active areas and wide Field-of-View (FoV) (Ref. 10).

A novel OWC solution developed for the AIT application will be presented in this paper. The system will be able to replace the MIL-STD-1553B cables by means of optical Transceivers (TRXs), which are realized exploiting low-cost and Commercial Off-the-Shelf (COTS) components. The military standard MIL-STD-1553B was first developed for aircraft, but in the last twenty years was seen a growing interest from the space industry in the use of MIL-STD-1553B instead of other standards. According to the standard specifications, its transmission is an half-duplex with a Manchester biphase codification and a data rate of $1 \mathrm{Mb} / \mathrm{s}$. These characteristics make the MIL-STD-1553B an optimal choice to be implemented with an OWC system.

\section{PROPOSED APPROACH}

The core of the proposed system is the TRX board, which was specially designed to meet the AIT scenario requirements. The system was designed in order to establish a bidirectional $\mathrm{OW}$ connection between the $\mathrm{S} / \mathrm{C}$ units under test and the Electrical Ground Support Equipment (EGSE), this link should be able to perform a data transmission replacing the cable connections, according to the the MIL-STD-1553B standard specifications.

The MIL-STD-1553B defines a digital time division command/response multiplexed data bus. Its data transmission uses a bit encoding according to a Manchester II biphase code and this signal is conveyed to a pair of cables as a differential voltage. Data transmission is performed using data packet defined words with a bitrate of $1 \mathrm{Mb} / \mathrm{s}$. We highlight that in an AIT scenario, the units under test are not in reciprocal visibility, i.e. there is no Line-of-Sight (LoS) between Transmitter (TX) and Receiver (RX). This requires that the transmission on the light between TX and RX takes place via secondary paths thanks to reflections on the ceiling or from other surfaces. In order to satisfy this requirement, the OWC link is designed according to a non-Line of Sight (n-LoS) architecture: we realized a TX with wide emission angle, able to establish an extremely robust connection with respect to the relative position of the RX. Our TRX is made of a TX, a RX and a Signal Adaptation Board (SAB), and is schematically represented in Fig. 1. 
The TX consists of an infrared Light Emitting Diode (IR-LED), used as optical source and a driver electrical circuit. The LED was chosen because it provides a light beam with the wide emission angle needed to allow the realization of a n-LoS configuration. The circuit is needed to drive properly the optical source, which emits in the near infrared range $(850-940 \mathrm{~nm})$. Silicon-based Photodiodes (PDs) have maximum responsivity in this spectral range. Moreover, could be convenient to cut-off the visible wavelengths (present in the AIT scenario) by an optical filter. The RX side is comprised of a P-I-N junction phododiode (PIN-PD) and a Transimpedance Amplifier (TIA), which amplifies and converts the current signal by the PIN-PD into a voltage signal. This class of photodiodes turn to be the best choice because of the large active area and the high FoV needed to increase the collected optical power. The third element of the TRX is the SAB. A MIL-STD-1553B differential signal cannot be straight transmitted using optical devices because of its features, e.g. the use of a 3 lines cable to run bus signal, all used for bidirectional transmission. Therefore, we designed a section of the TRX to allow the transmission of MIL-STD-1553B signal over OWC link. The SAB performs the conversion between the differential bipolar signal on the MIL-STD-1553B Data Bus to an unipolar single-ended signal needed for the OWC transmission; this conversion acts on the amplitude levels of the signal, keeping the packet structure of the signal unaltered, allowing for a power consumption reduction. According to MIL-STD-1553B specifications, the data bus is used for both transmitting and receiving data. The SAB is able to recognize the correct data flow direction and convey signals on TX or RX, depending on the transmission mode, without any type of digital processing devices (e.g., FPGA or microprocessors). The last task performed by the SAB is the creation of a time windows to disable RX section while TX is transmitting, both belonging to the same TRX; this avoids optical cross-talk, which may arise in case of back-reflections.

The MIL-STD-1553B differential signal from the bus is sent to a first TRX and converted by the SAB into a single-ended signal unipolar 0-5 V. The transmitter driver converts this single-ended signal into a current that drives the optical source, which is then turned OFF and ON. The driver is mainly required for the n-LoS TRX since the power from the level adaptation output is not enough to drive the LEDs. The optical signal is received by the RX board of a second TRX. The PIN-PD transduces the optical signal to current by photoelectric effect and sends it to the TIA to be amplified and converted into a voltage signal. The TIA circuitry is designed to be AC-coupled to filter out ambient light and to avoid the saturation of the amplifiers. Other electronic components recover the data with a suitable voltage amplitude (0-5 V) to be fed back to the SAB. In the end, the single-ended signal is converted again to a MIL-STD-1553B differential signal and is sent to the MIL-STD-1553B bus. During the transmission, the RX belonging to the first TRX is kept disabled to avoid optical cross-talk effects.

\section{EXPERIMENTAL SETUP AND RESULTS}

In this section, we describe the experimental setup used to carry out the OWC system characterization and we report and discuss the obtained experimental results .

The proposed system was designed, implemented and tested in our laboratory. The tested TRX was realized using a IR-LED with central wavelength of $850 \mathrm{~nm}$ and an emission angle of $150^{\circ}$, for the TX and a PIN-PD with a photosensitive area of $26.4 \mathrm{~mm}^{2}$, a maximum responsivity at $950 \mathrm{~nm}$ and a FoV of $120^{\circ}$, for the RX. To simulate the MIL-STD-1553B bus, the transmitting signal was generated by means of a Test \& Simulation USB module (Test Module (TM)) by Avionics Interface Technologies, compliant with the MIL-STD-1553B standard. To perform these test, a 3 words packet was used for a total time length of $60 \mu$ s for each packet.

A TM was connected to the first TRX and another one was used to receive the signal from the second TRX, both the TMs were connected and driven by the Avionics Interface Technologies software. Using a C\# homemade routine for transmission and detection, Bit Error Ratio (BER) measurement was performed. A picture of the experimental setup is reported in Fig. 2.

These measurements were performed to verify the TRX performance in common conditions provided for the AIT operations. During typical AIT tests, the equipment was placed on a table and connected to the system bus, whereas the EGSE equipement is connected to the system bus as well. Therefore, the measurements were performed according to this AIT scenario and 3 different configurations were identified. These configurations are summarized in Fig. 3. The setup was designed and implemented at the laboratories of Scuola Superiore Sant'Anna, to reproduce the characteristics of the AIT scenario as faithfully as possible. In configuration 1 and 


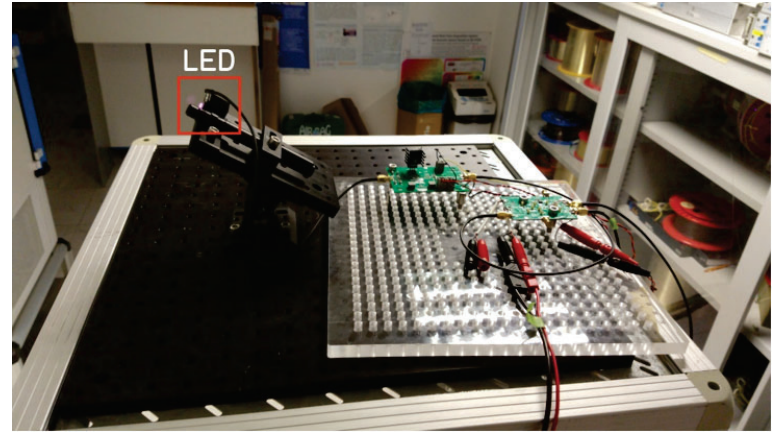

(a)

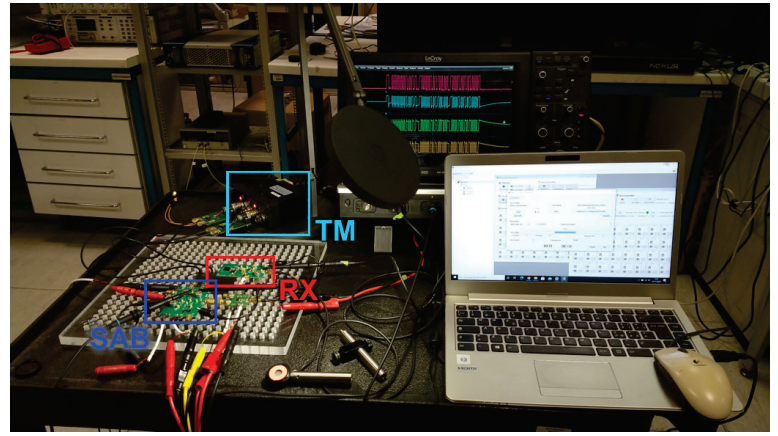

(b)

Figure 2: Experimental setup. (a) TX: LED and driver, (b) RX: PD+TIA, SAB and a TM

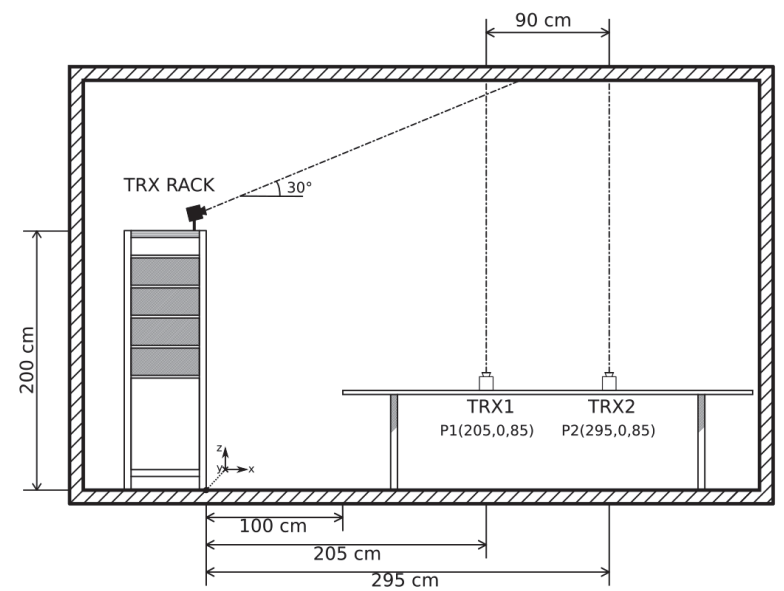

(a)

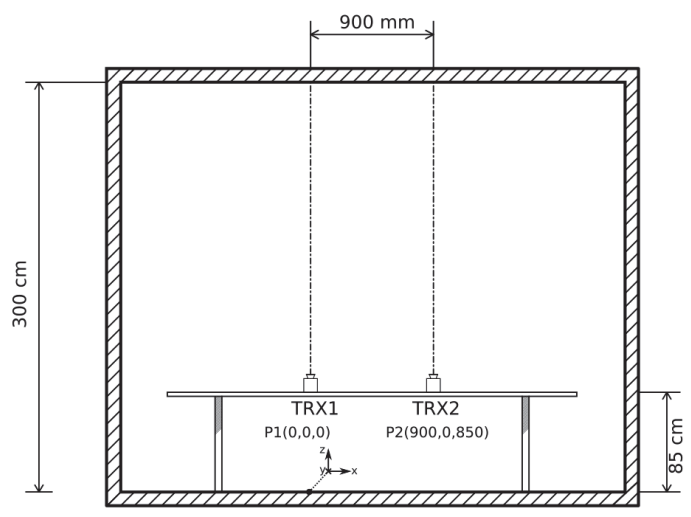

(b)

Figure 3: Measurement scenarios

2 (Fig. 3a), the TRX is placed on the top of the EGSE (TRX RACK in Fig. 3a) at $2 \mathrm{~m}$ from the ground, mounted on a platform tilted by $30^{\circ}$. A second TRX (TRX1 and TRX2 in Fig. 3a) in placed on a bench, at 85 $\mathrm{cm}$ from the ground, alternatively in position $\mathrm{P} 1$ or $\mathrm{P} 2$, at $205 \mathrm{~cm}$ and $295 \mathrm{~cm}$ from the rack respectively. The tilting of the mounting platform is needed to realize a n-LoS transmission exploiting reflections on the ceiling 3 $\mathrm{m}$ height. These two configurations differ in the direction of transmission:

- configuration 1, TRX RACK is transmitting and TRX1/TRX2 is receiving;

- configuration 2, TRX RACK is receiving and TRX1/TRX2 is transmitting;

In configuration 3, both TRXs are placed on the bench, TRX1 is transmitting and TRX2 is receiving. In all three scenarios, a cold-white LED lamp was used to control the luminous flux on the RX; an illuminance of around $700 \mathrm{~lx}$ was guaranteeded to simulate standard conditions in a typical working environment. The illuminance measurements was performed by means of the Luxmeter Sekonic Spectromaster C-700, placed next to the RX.

\subsection{RX Sensitivity Measurements}

As preliminary test, the RX sensitivity was measured using configuration 3. The sensitivity defines the optical power required to get a given BER value (here $10^{-12}$ ). This value is needed to define the performance of a $\mathrm{RX}$ and it is a key parameter for the characterization of the TRX. Therefore, this result will be used to have a reference value for our OWC performance in the AIT scenario. The sensitivity measurements were performed 


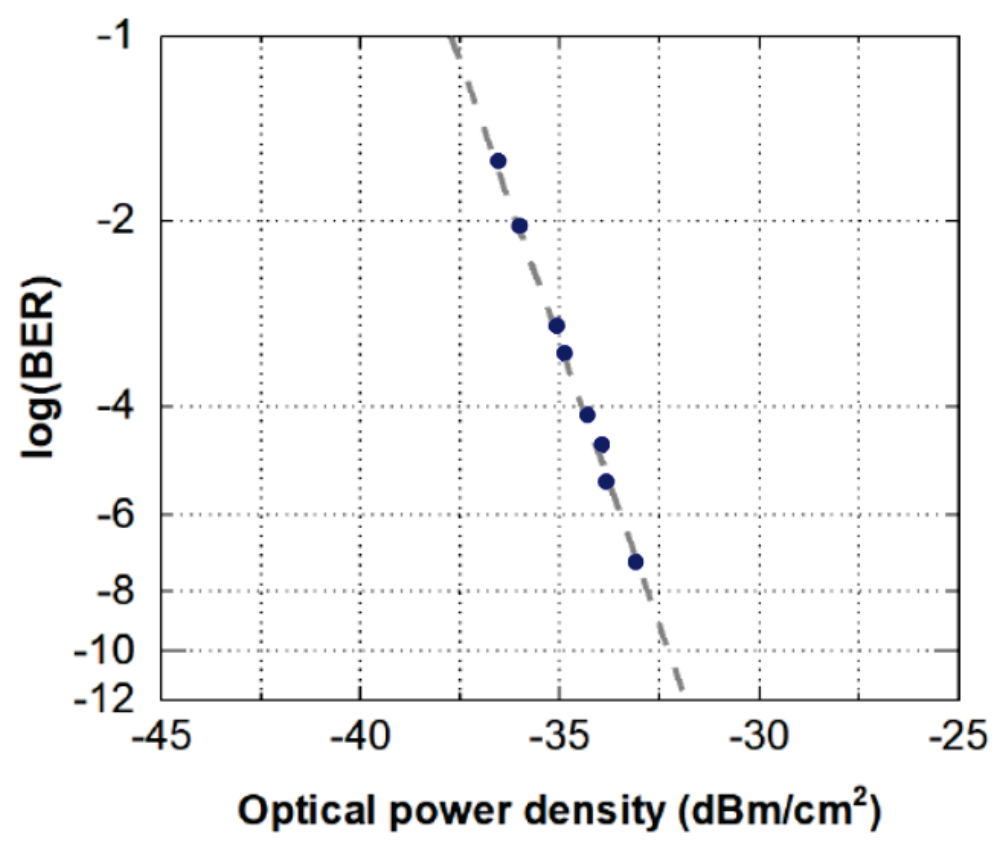

Figure 4: RX sensitivity curve for MIL-STD-1553B signal. BER measurements are plotted as a function of the received optical power $P_{d}$

varying the received optical power density $\left(P_{d}\right)$ on the PD using optical attenuators placed in front of the TX. Changing the $P_{d}$ at the receiver in a range between $-28 \mathrm{dBm} / \mathrm{cm}^{2}$ and $-36 \mathrm{dBm} / \mathrm{cm}^{2}$, the BER curve was obtained and the receiver sensitivity estimated. The resulting BER curve is shown in Fig. 4; on the X-axis we report the received $P_{d}$, whereas on the Y-axis we report $\log (\mathrm{BER})$. The results are expressed in $\mathrm{dBm} / \mathrm{cm}^{2}$ to make them independent from the RX effective area and position in the scenario.

A BER lower than $10^{-8}$ cannot be detected because of the low available bitrate $(40 \mathrm{~kb} / \mathrm{s}$ ), this low bitrare is mainly due to the difference between the idle and packets time durations. Moreover an off line data analysis was performed, further reducing the effective bitrate. However, according to theory, it is possible to estimate the required BER from a linear fit of the logarithm of the measured BER values and then extrapolate to get the $P_{d}$ needed to have a $B E R=10^{-12}$. The $P_{d}$ represents the RX sensitivity (considering a BER target of $10^{-12}$ ) and it is equal to $-32 \mathrm{dBm} / \mathrm{cm}^{2}$.

\subsection{AIT Measurements}

In this section, we report the results of the AIT tests. These were performed for configurations 1 in $\mathrm{P} 1$ and P2, configuration 2 in P1 and P2 and configuration 3, as previously defined.

In the first configuration the transmission direction is from the rack to the bench, as shown in Fig. 3a. The TRX RACK is transmitting and TRX1/TRX2 is receiving, first in position P1 and then in P2. The received electrical signal from RX in positions P1 and P2, and the corresponding eye diagrams are reported in Fig. 5 and Fig. 6, respectively. As can be seen from the eye diagram in Fig. 5b and 6b, the signal is perfectly received. The measured $P_{d}$ is $-28.3 \mathrm{dBm} / \mathrm{cm}^{2}$ in position $\mathrm{P} 1,-31 \mathrm{dBm} / \mathrm{cm}^{2}$ in position $\mathrm{P} 2$. In both positions the $P_{d}$ results to be higher than the estimated RX sensitivity at $B E R=10^{-12}$, with a link margin of $4 \mathrm{~dB}$ in $\mathrm{P} 1$ and $1 \mathrm{~dB}$ in $\mathrm{P} 2$. The measured BER is lower than $10^{-8}$, i.e. after a transmission of $10^{8}$ no errors were observed.

In the second configuration, the transmission direction is from the bench to the rack, as shown in Fig. 3a. The TRX RACK is receiving and TRX1/TRX2 is transmitting, alternatively in position P1 and P2. The received electrical signal for TX in position P1 and P2, and the corresponding eye diagrams are reported in Fig. 7 and Fig. 8, respectively. As can be seen, the signal is perfectly received. The measured $P_{d}$ is $-27.6 \mathrm{dBm} / \mathrm{cm}^{2}$ in position $\mathrm{P} 1$ and $-29.6 \mathrm{dBm} / \mathrm{cm}^{2}$ in position $\mathrm{P} 2$. In both positions the $P_{d}$ values is higher than the estimated RX 


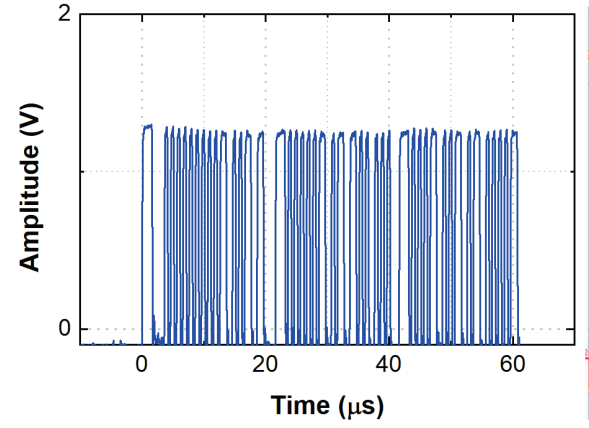

(a)

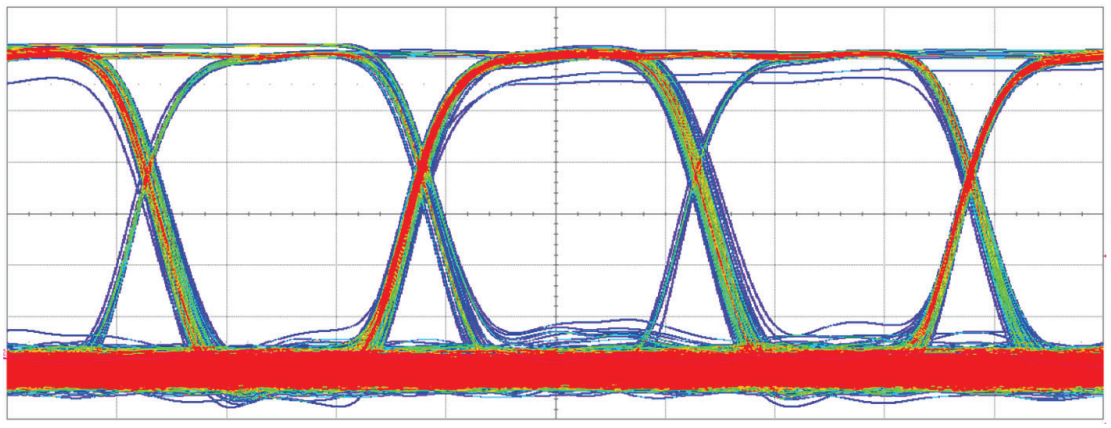

(b)

Figure 5: Electrical signal (a) and electrical eye diagram (b) at the output of the receiver in configuration 1, RX in P1

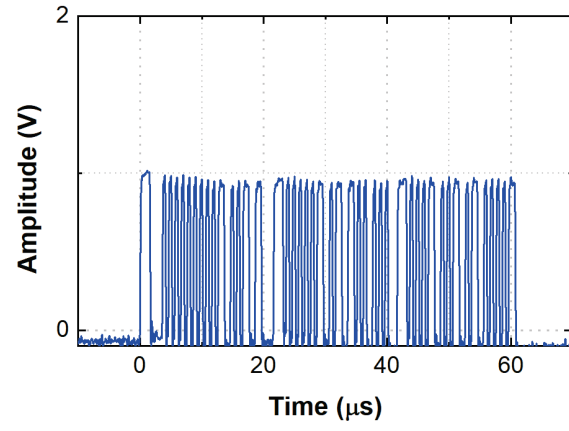

(a)

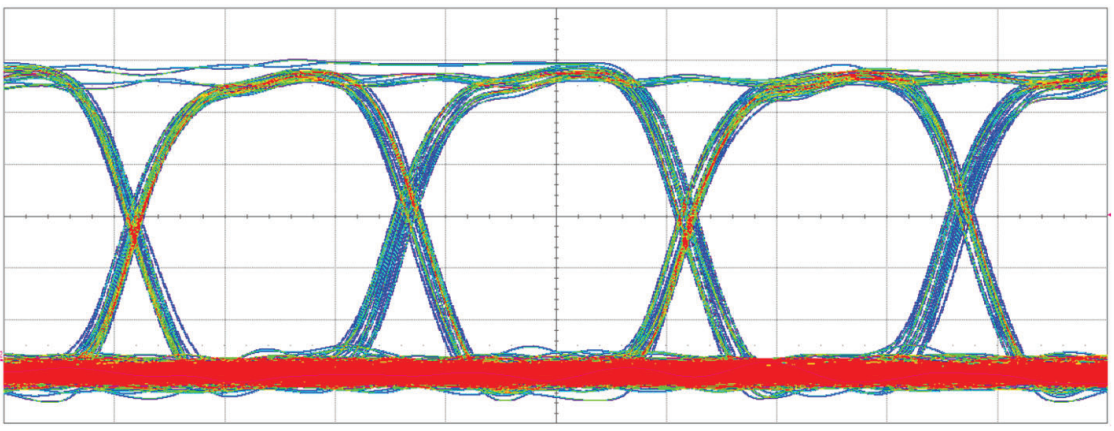

(b)

Figure 6: Electrical signal (a) and electrical eye diagram (b) at the output of the receiver in configuration 1, RX in P2

sensitivity, with a link margin of $4.4 d B$ in $\mathrm{P} 1$ and $2.4 \mathrm{~dB}$ in $\mathrm{P} 2$. The measured BER is lower than $10^{-8}$, i.e. after transmission of $10^{8}$ no errors were observed.

In the last configuration, the TRXs are placed on two benches, as presented in Fig. 3b and the transmission direction is from TRX1 to TRX2. The received electrical signal from RX and the corresponding eye diagram are reported in Fig. 9. Also in this case, we see that the signal is perfectly received. The measured $P_{d}$ is -28.2 $\mathrm{dBm} / \mathrm{cm}^{2}$ and is higher than the RX sensitivity, with a link margin of $3.8 \mathrm{~dB}$. The measured BER is lower than

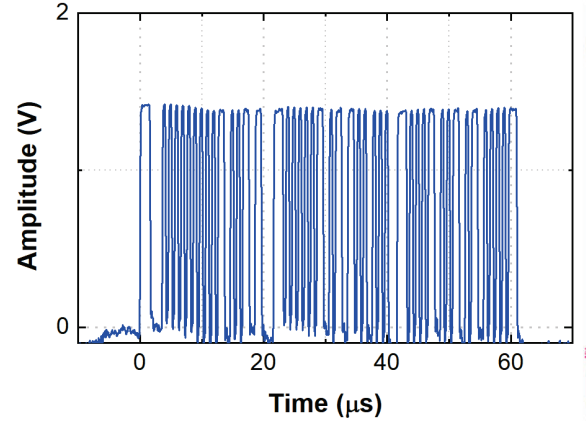

(a)

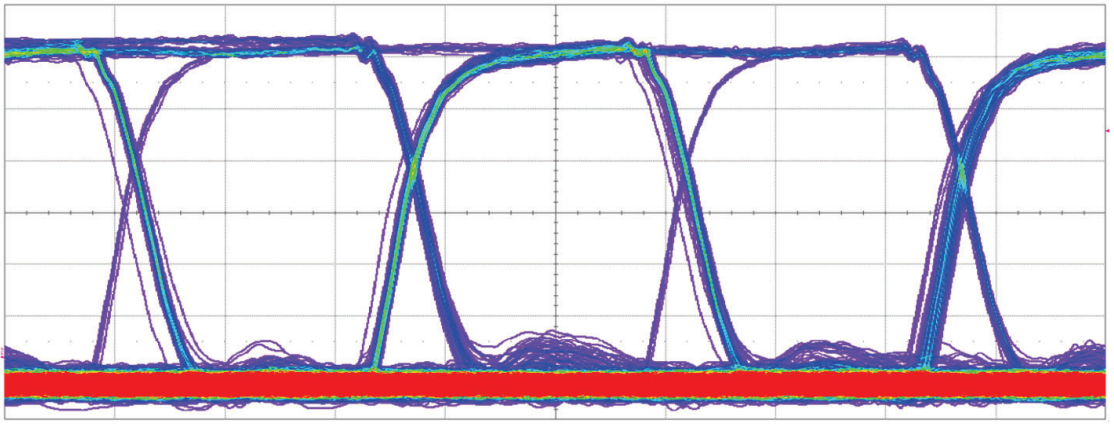

(b)

Figure 7: Electrical signal (a) and electrical eye diagram (b) at the output of the receiver in configuration 2, RX in $\mathrm{P} 1$ 


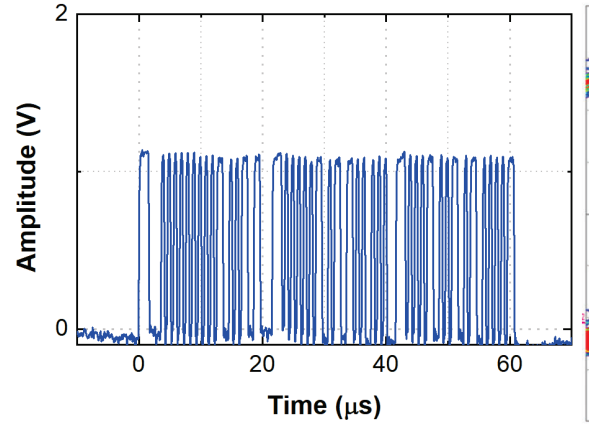

(a)

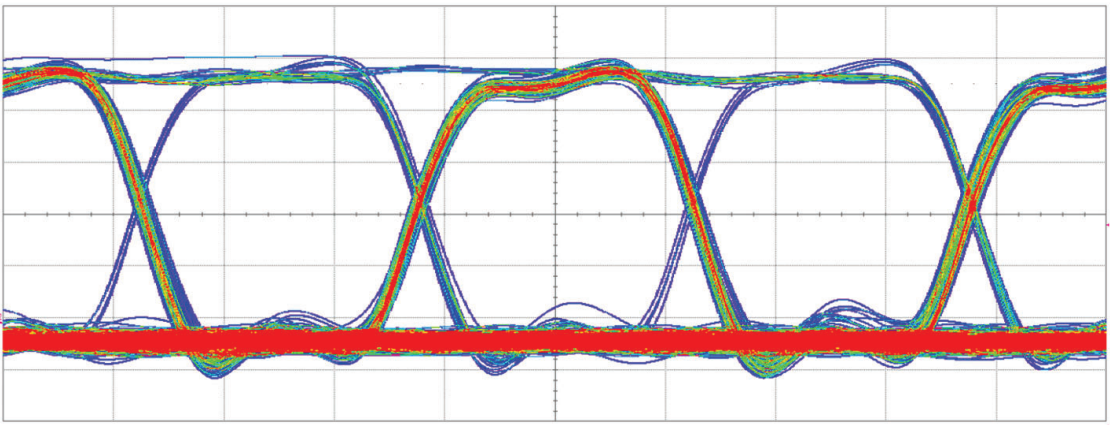

(b)

Figure 8: Electrical signal (a) and electrical eye diagram (b) at the output of the receiver in configuration 2, RX in $\mathrm{P} 2$

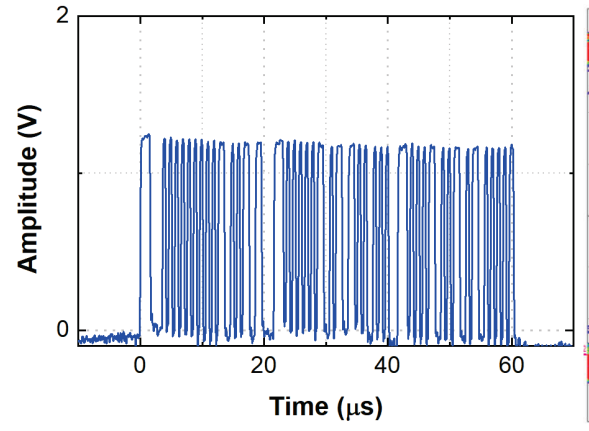

(a)

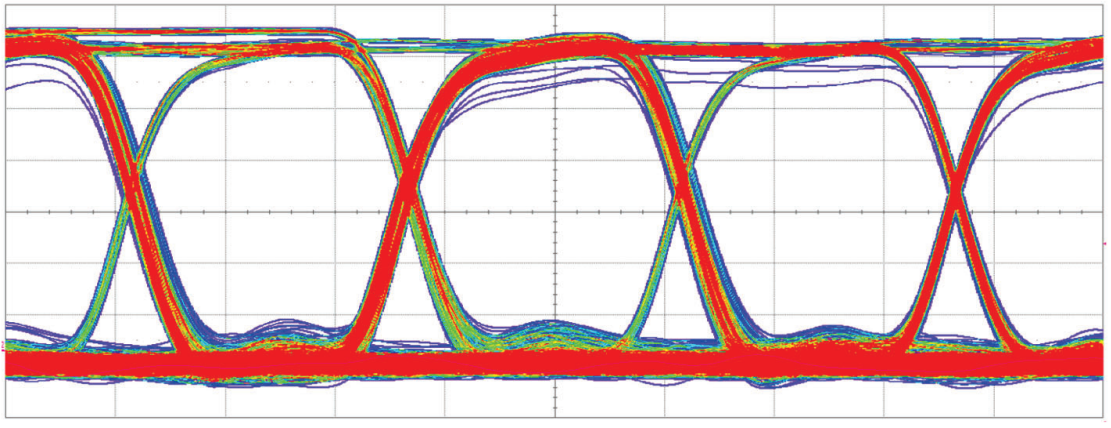

(b)

Figure 9: Electrical signal (a) and electrical eye diagram (b) at the output of the receiver in configuration 3

$10^{-8}$, i.e. after a transmission of $10^{8}$ no errors were observed.

This set of results is summarized in table 1 . As it can be seen, in the three configurations a received $P_{d}$ higher than the RX sensitivity $\left(-32 \mathrm{dBm} / \mathrm{cm}^{2}\right)$ was measured and no transmission error was detected. The estimated link margins are sufficiently high to consider the link stable. Only in the configuration 1 P2 the link margin is $1 \mathrm{~dB}$, this makes this configuration the least robust. Based on the achieved results, the OWC system is an excellent candidate to substitute MIL-STD-1553B cable connections in AIT phase.

Table 1: Summary AIT tests results for the three configurations

\begin{tabular}{|c|c|c|c|}
\hline Configuration & Measured BER & $P_{d} \mathrm{dBm} / \mathrm{cm}^{2}$ & Link Margin $(\mathrm{dB})$ \\
\hline $1 \mathrm{P} 1$ & $<10^{-8}$ & -28.3 & 4 \\
\hline $1 \mathrm{P} 2$ & $<10^{-8}$ & -31 & 1 \\
\hline $2 \mathrm{P} 1$ & $<10^{-8}$ & -27.6 & 4.4 \\
\hline $2 \mathrm{P} 2$ & $<10^{-8}$ & -29.6 & 2.4 \\
\hline 3 & $<10^{-8}$ & -28.2 & 3.8 \\
\hline
\end{tabular}

\section{CONCLUSION}

A new OWC system design in a typical AIT scenario was presented and demonstrated. The system implements a bidirectional communication between S/C devices and the EGSE, able to replace the standard MIL-STD-1553B 
cables. These results were achieved by means of OWC transceivers that use a IR-LED as optical source and a PIN-PD as optical receiver. All the optical and electronic systems were realized exploiting low-cost and COTS components. The estimated RX sensitivity, was of $-32 \mathrm{dBm} / \mathrm{cm}^{2}$ (BER of $10^{-12}$ ). A first characterization of the TRX was performed in the laboratory, emulating a real AIT environment. Here, the performance was tested in three different cases. In each configuration, a MIL-STD-1553B signal was transmitted and the BER and the received $P_{d}$ were measured to calculate the link margin. All the obtained results show that the OWC system is compatible with the transmission requirements in the AIT scenario. In conclusion, these first results demonstrate that the OWC systems are a promising candidate to replace the cable connection in AIT operations and that can be integrated as $\mathrm{S} / \mathrm{Cs}$ communication systems.

\section{ACKNOWLEDGMENT}

This work was partly supported by ESA Project TOWS (Contract 4000125458/18/UK/NR).

\section{REFERENCES}

[1] Jablonski, A. and Showalter, D., "Recent Advances in Assembly, Integration, and Testing (AIT) at the David Florida Laboratory," (05 2013).

[2] CCSDS, "Wireless network communications overview for space missions operations." Green Book, May 2017 https://https://public.ccsds.org/Pubs/880x0g3.pdf.

[3] Ratiu, O., Rusu, A., Pastrav, A., Palade, T., and Puschita, E., "Implementation of an UWB-based module designed for wireless intra-spacecraft communications," in [2016 IEEE International Conference on Wireless for Space and Extreme Environments (WiSEE)], 146-151 (2016).

[4] Sidibeh, K. and Vladimirova, T., "wireless communication in leo satellite formations," in [2008 NASA/ESA Conference on Adaptive Hardware and Systems],

[5] Arruego, I., Guerrero, M. T., Rodriguez, S., Martinez-Oter, J., Jimenez, J. J., Dominguez, J. A., MartinOrtega, A., de Mingo, J. R., Rivas, J., Apestigue, V., Sanchez, J., Iglesias, J., Alvarez, M. T., Gallego, P., Azcue, J., Ruiz de Galarreta, C., Martin, B., Alvarez-Herrero, A., Diaz-Michelena, M., Martin, I., Tamayo, F. R., Reina, M., Gutierrez, M. J., Sabau, L., and Torres, J., "OWLS: a ten-year history in optical wireless links for intra-satellite communications," IEEE Journal on Selected Areas in Communications 27(9), 15991611 (2009).

[6] Ciaramella, E., Cossu, G., Ertunc, E., Gilli, L., Messa, A., Rannello, M., Presi, M., Sturniolo, A., Bresciani, F., and Podda, V., "Tows: Introducing optical wireless for satellites," in [2019 21st International Conference on Transparent Optical Networks (ICTON)], 1-4 (2019).

[7] Ali, W., Cossu, G., Gilli, L., Ertunc, E., Messa, A., Sturniolo, A., and Ciaramella, E., "10 Gbit/s OWC System for Intra-Data Centers Links," IEEE Photonics Technology Letters 31(11), 805-808 (2019).

[8] Hamza, A. S., Deogun, J. S., and Alexander, D. R., "Wireless communication in data centers: A survey," IEEE Communications Surveys Tutorials 18(3), 1572-1595 (2016).

[9] Cossu, G., Ali, W., Rannello, M., Ertunc, E., Gilli, L., Sturniolo, A., Messa, A., and Ciaramella, E., "VCSEL-Based 24 Gbit/s OWC Board-to-Board System," IEEE Communications Letters 23(9), 1564-1567 (2019).

[10] Komine, T. and Nakagawa, M., "Fundamental analysis for visible-light communication system using led lights," IEEE Transactions on Consumer Electronics 50(1), 100-107 (2004). 